\title{
Feedback Numbers of Möbius Ladders
}

\author{
Yijin Wang ${ }^{1}$, Xinyue Zhang $^{1}$ and Sijia Zhang $^{1, a}$ \\ ${ }^{1}$ School of Information Engineering, Dalian Ocean University, Dalian, 116023, China
}

\begin{abstract}
A subset $F \subset V(G)$ is called a feedback vertex set if the subgraph $\mathrm{G}-\mathrm{F}$ is acyclic. The minimum cardinality of a feedback vertex set is called the feedback number of $\mathrm{G}$, which is proposed by Beineke and Vandell [1]. In this paper, we consider a particular topology graph called Möbius ladders $\mathrm{M}_{2 \mathrm{n}}$. We use $f\left(\mathrm{M}_{2 \mathrm{n}}\right)$ to denote the feedback number of $\mathrm{M}_{2 \mathrm{n}}$. This paper proves that $f\left(M_{2 n}\right)=\left[\frac{n+1}{2}\right], n \geq 3$.
\end{abstract}

\section{Introduction}

It is well-known that the underlying topology of an interconnection network can be represented by a connected graph $G=(V, E)$, where $V$ is the set of processors and $E$ is the set of communication links in the network. In this paper, we use graphs and interconnection networks interchangeably.

Let $\mathrm{G}=(\mathrm{V}, \mathrm{E})$ be a graph or digraph without multiple edges, with vertex set $\mathrm{V}(\mathrm{G})$ and edge set $\mathrm{E}(\mathrm{G})$. A subset $F \subset V(G)$ is called a feedback vertex set if the subgraph G-F is acyclic, that is, if G-F is a forest. The minimum cardinality of a feedback vertex set is called the feedback number (or decycling number proposed first by Beineke and Vandell [1]) of $G$. A feedback vertex set of this cardinality is called a minimum feedback vertex set.

Apart from its graph-theoretical interest, the minimum feedback vertex set problem has important application to several fields. For example, the problems are in operating systems to resource allocation mechanisms that prevent deadlocks [2], in artificial intelligence to the constraint satisfaction problem and Bayesian inference, in synchronous distributed systems to the study of monopolies and in optical networks to converters placement problem (see [3, 4]).

Determining the feedback number is quite difficult even for some elementary graphs. However, the problem has been studied for some special graphs and digraphs, such as hypercubes, meshes, to roids, butterflies, cubeconnected cycles, directed split-stars (see $[3,4,5,6,7,8$, $9,10,11,12,13])$. In fact, the minimum feedback set problem is known to be NP - hard for general graphs [14] and the best known approximation algorithm is one with an approximation ratio two [5].

In this paper, we consider the feedback number of Möbius Ladders $M_{2 n}$. Let $f\left(M_{2 n}\right)$ denote the feedback number of Möbius ladders. By using an approach to construct an acyclic subgraph of $M_{2 n}$, we proves that:

$$
f\left(M_{2 n}\right)=\left\lceil\frac{n+1}{2}\right\rceil n \geq 3 .
$$

\section{Preliminary}

R. L.Guy and F. Harary consider the graph $M_{\mathrm{L}}$ (also called the Möbius ladder) in1966, which we picture as a ladder with the ends joined after a twist, so that the graph is naturally embedded in a Möbius band [16].
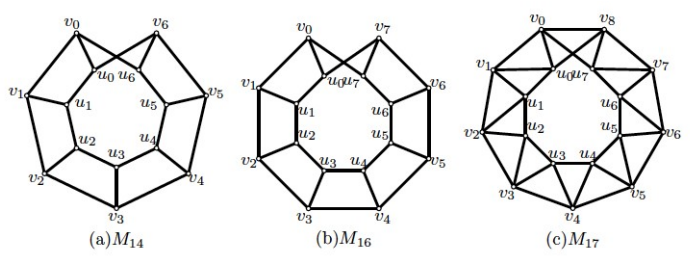

Figure 1. Examples of Möbius ladders

One particular molecular graph which is of interest is the molecular Möbius ladder, which was first synthesized by Walba et al. It was a significant achievement in chemistry because of its topologically interesting molecular structure. Simon proved that its associated embedded molecular graph was topologically chiral [17].

Although many authors only consider even L, but there exists odd L which be mentioned in ref. [18]. And as evidenced by the number of publications, there are many more established results for even L Möbius ladders than for odd. Obviously, the two cases (L even or odd) change the structure of these graphs quite radically:

Case1. When $\mathrm{L}$ is even, $M_{L}$ is three-regular and Hamiltonian (See Fig.1(a)-(b)).

Case2. When $\mathrm{L}$ is odd, $M_{L}$ is four-regular and therefore two-factorable (See Fig.1(c)).

\footnotetext{
a Corresponding author: zhangsijia@dlou.edu.cn
} 
In this paper, we study only Case 1, and only even $L \geq 6$. Unless otherwise stated, assume that $M_{L}$ means an even Möbius Ladder.

Definition 2.1. A Möbius Ladder is defined to be a graph on $2 n(n \geq 3)$ vertices with

$$
V\left(M_{2 n}\right)=\left\{v_{i}, u_{i}: 0 \leq i \leq n-1\right\}
$$

and

$$
\begin{aligned}
E\left(M_{2 n}\right) & =\left\{v_{i} u_{i}: 0 \leq i \leq n-1\right\} \\
& \cup\left\{v_{i} v_{i+1}, u_{i} u_{i+1}: 0 \leq n-1\right\} \\
& \cup\left\{u_{n-1} v_{0}, v_{n-1} u_{0}\right\}
\end{aligned}
$$

Obviously, $M_{2 n}$ is three-regular and Hamiltonian.

\section{Feedback vertex set of $M_{2 n}$}

Since $N=2 n$, let $n \bmod 2=k$, then $n=2 m+k, m \geq 1$ and $k \in[0,1]$.

Let $V_{a}^{1}\left(M_{2 n}\right)=F_{1}^{n} \cup F_{2}^{n} \subset V\left(M_{2 n}\right)$, then we discuss two cases as follows.

Case 1. If $n \bmod 2=1$ and odd $n \geq 3$, let

$$
\begin{aligned}
& F_{1,1}^{n}=\bigcup_{\substack{i=0, i \bmod 2=0}}^{n-1}\left\{v_{i}\right\} \cup \bigcup_{\substack{i=0, i \bmod 2=1}}^{n-2}\left\{u_{i}\right\} \\
& F_{1,2}^{n}=\bigcup_{\substack{i=0, i \bmod 2=1}}^{n-2}\left\{v_{i}\right\}
\end{aligned}
$$

Case 2. If $n \bmod 2=0$ and even $n \geq 4$, let

$$
\begin{aligned}
& F_{2,1}^{n}=\bigcup_{\substack{i=0, i \bmod 2=0}}^{n-1}\left\{v_{i}\right\} \cup \bigcup_{\substack{i=0, i \bmod 2=1}}^{n-2}\left\{u_{i}\right\} \\
& F_{2,2}^{n}=\bigcup_{\substack{i=0 \\
i \bmod 2=1}}^{n-2}\left\{v_{i}\right\} \\
& F_{2,3}^{n}=\left\{u_{n-1}\right\}
\end{aligned}
$$

Definition 3.1. The open neighborhood and the closed neighborhood of a vertex $v \in V\left(M_{2 n}\right)$ are denoted by $N(v)=\left\{u \in V\left(M_{2 n}\right): v u \in E\left(M_{2 n}\right)\right\}$

and $N[v]=N(v) \cup\{v\}$, respectively. For a vertex set $S \subseteq V\left(M_{2 n}\right), N(S)=\bigcup_{v \in S} N(v)$ and $N[S]=\bigcup_{v \in S} N[v]$.

Definition 3.2. For any vertex $u_{j} \in F_{i, 2}^{2}, i \in[1,2]$ and $j \in[0, n-1]$, denote $T_{1, j}=N\left[u_{j}\right]$, where $N\left[u_{j}\right]$ is the closed neighborhood of a vertex $u_{j}$ and the induced subgraph of $T_{1, j}$ as $G\left[T_{1, j}\right]$.

In fact, for any $j, G\left[T_{1, j}\right]$ is isomorphic to $K_{1,3}$.That is, for any $j, G\left[T_{1, j}\right]$ is a tree, where vertex $v_{j}$ is a root vertex of tree $G\left[T_{1, j}\right]$ and vertex set $N\left(v_{j}\right)$ are leaf vertices of tree $G\left[T_{1, j}\right]$.

By the Definition 2.1, all the vertices in $F_{i, 1}^{n}$ and $F_{i, 2}^{n}$ are isolated vertex set for $i \in[1,2]$, respectively. Then the induced subgraph of $F_{i, 1}^{n}$ is acyclic and the induced sub-graph of $F_{i, 2}^{n}$ is acyclic. By the Definition 3.2, we obtain $N\left(F_{i, 2}^{n}\right)=F_{i, 1}^{n}$.

Lemma 3.1. $G\left[V_{a}^{1}\left(M_{2 n}\right)\right]$ is acyclic for $n \bmod 2=1$ and odd $n \geq 3$.

Proof.

Since

$$
\begin{aligned}
V_{a}^{1}\left(M_{2 n}\right) & =F_{1,1}^{n} \cup F_{1,2}^{n} \\
& =N\left(F_{1,2}^{n}\right) \cup F_{1,2}^{n} \\
& =N\left[F_{1,2}^{n}\right]
\end{aligned}
$$

Then if we want to prove $G\left[V_{a}^{1}\left(M_{2 n}\right)\right]$ is acyclic, which is equivalent to verity that $G\left[N\left[F_{1,2}^{n}\right]\right]$ is acyclic.

Firstly, we prove that $G\left[N\left[F_{1,2}^{n}\right]\right]$ is a tree by induction on $n$.

We first prove the basic step for $n=3$.

Since

$$
F_{1,1}^{3}=\left\{v_{0}, v_{2}\right\} \cup\left\{u_{1}\right\}, F_{1,2}^{3}=\left\{v_{1}\right\}
$$

And

$$
\begin{aligned}
T_{1,1}^{3} & =N\left[v_{1}\right]=N\left\{v_{1}\right\} \cup\left\{v_{1}\right\} \\
& =\left\{v_{0}, v_{2}\right\} \cup\left\{v_{1}\right\} \\
& =F_{1,1}^{3} \cup F_{1,2}^{3} \\
& =N\left[F_{1,2}^{3}\right]
\end{aligned}
$$

Then $G\left[T_{1,1}^{3}\right]$ is a tree. That is, $G\left[F_{1,1}^{3} \cup F_{1,2}^{3}\right]$ is a tree.

Suppose $G\left[F_{1,1}^{n} \cup F_{1,2}^{n}\right]$ is a tree, we now prove that $G\left[F_{1,2}^{n+2} \cup F_{1,2}^{n+2}\right]$ is a tree.

Since

$$
\begin{aligned}
F_{1,1}^{n+2} & =\bigcup_{\substack{i=0, i \bmod 2=0}}^{n+2-1}\left\{v_{i}\right\} \cup \bigcup_{\substack{i=0, i \bmod 2=1}}^{n+2-2}\left\{u_{i}\right\} \\
& =\bigcup_{\substack{i=0, i \bmod 2=0}}^{n-1}\left\{v_{i}\right\} \cup \bigcup_{\substack{i=n, i \bmod 2=0}}^{n+1}\left\{v_{i}\right\} \cup \bigcup_{\substack{i=0, i \bmod 2=1}}^{n-2}\left\{u_{i}\right\} \cup \bigcup_{\substack{i=n-1, i \bmod 2=1}}^{n}\left\{u_{i}\right\} \\
& =F_{1,1}^{n} \cup \bigcup_{\substack{i=n \\
i \bmod 2=0}}^{n+1}\left\{v_{i}\right\} \cup \bigcup_{i=n-1,}^{n}\left\{u_{i}\right\} \\
& =F_{1,1}^{n} \cup\left\{v_{n+1}\right\} \cup\left\{u_{n}\right\}
\end{aligned}
$$


and

$$
\begin{aligned}
F_{1,2}^{n+1} & =\bigcup_{\substack{i=0, i \bmod 2=1}}^{n+2-2}\left\{v_{i}\right\} \quad=\bigcup_{\substack{i=0, i \bmod 2=1}}^{n-2}\left\{v_{i}\right\} \cup \bigcup_{\substack{i=n-1, i \bmod 2=1}}^{n}\left\{v_{i}\right\} \\
& =F_{1,2}^{n} \cup\left\{v_{n}\right\}
\end{aligned}
$$

Then

$$
\begin{aligned}
F_{1,1}^{n+2} \cup F_{1,2}^{n+2} & =F_{1,1}^{n} \cup F_{1,2}^{n} \cup\left\{v_{n+1}, u_{n}, v_{n}\right\} \\
& =F_{1,1}^{n} \cup F_{1,2}^{n} \cup T_{1, n}^{n+2}
\end{aligned}
$$

Since $G\left[T_{1, n}^{n+2}\right] \cong K_{1,3}$, then $G\left[T_{1, n}^{n+2}\right]$ is a tree.

By the induction hypothesis, $G\left[F_{1,1}^{n} \cup F_{1,2}^{n}\right]$ is a tree.

Tree $G\left[T_{1, n}^{n+2}\right]$ and tree $G\left[F_{1,1}^{n} \cup F_{1,2}^{n}\right]$ just have one common leaf vertex $v_{n-1}$, then $G\left[F_{1,1}^{n} \cup F_{1,2}^{n} \cup T_{1, n}^{n+2}\right]$ is a tree.

Combining induction step with basic step,

$G\left[F_{1,2}^{n} \cup F_{1,2}^{n}\right]$ is a tree.

Thus, $G\left[V_{a}^{1}\left(M_{2 n}\right)\right]=G\left[F_{1,1}^{n} \cup F_{1,2}^{n}\right]$ is acyclic.

The lemma holds.

Lemma 3.2. $G\left[V_{a}^{2}\left(M_{2 n}\right)\right]$ is acyclic for $n \bmod 2=0$ and even $n \geq 4$.

Proof.

Since

$$
\begin{aligned}
V_{a}^{2}\left(M_{2 n}\right) & =F_{2,1}^{n} \cup F_{2,2}^{n} \cup F_{2,3}^{n} \\
& =N\left(F_{2,2}^{n}\right) \cup F_{2,2}^{n} \cup\left\{u_{n-1}\right\} \\
& =N\left[F_{2,2}^{n}\right] \cup\left\{u_{n-1}\right\}
\end{aligned}
$$

Then if we want to prove $G\left[V_{a}^{2}\left(M_{2 n}\right)\right]$ is acyclic, which is equivalent to verity that $G\left[N\left[F_{2,2}^{n}\right] \cup\left\{u_{n-1}\right\}\right]$ is acyclic.

Firstly, we prove that $G\left[N\left[F_{2,2}^{n}\right] \cup\left\{u_{n-1}\right\}\right]$ is a tree by in-duction on $n$.

We first prove the basic step for $n=4$.

Since

$$
F_{2,1}^{4}=\left\{v_{0}, v_{2}\right\} \cup\left\{u_{1}\right\}, F_{2,2}^{4}=\left\{v_{1}\right\}, F_{2,3}^{4}=\left\{u_{3}\right\}
$$

And

$$
\begin{aligned}
T_{2,1}^{4} & =N\left[v_{1}\right]=N\left(v_{1}\right) \cup\left\{v_{1}\right\}=\left\{v_{0}, v_{2}, u_{1}\right\} \cup\left\{v_{1}\right\} \\
& =F_{2,1}^{4} \cup F_{2,2}^{4}=N\left[F_{2,2}^{2}\right]
\end{aligned}
$$

Since $G\left[T_{2,1}^{4}\right] \cong K_{1,3}$, then $G\left[T_{2,1}^{4}\right]$ is a tree. That is, $G\left[F_{2,1}^{4} \cup F_{2,2}^{4}\right]$ is a tree.

Suppose $G\left[F_{1,2}^{n} \cup F_{1,2}^{n}\right]$ is a tree, we now prove that $G\left[F_{1,2}^{n+2} \cup F_{1,2}^{n+2}\right]$ is a tree.

Since

$$
\begin{aligned}
F_{1,1}^{n+2} & =\bigcup_{\substack{i=0, i \bmod 2=0}}^{n+2-1}\left\{v_{i}\right\} \cup \bigcup_{\substack{i=0, i \bmod 2=1}}^{n+2-2}\left\{u_{i}\right\} \\
& =\bigcup_{\substack{i=0 \\
i \bmod 2=0}}^{n-1}\left\{v_{i}\right\} \cup \bigcup_{\substack{i=n, i \bmod 2=0}}^{n+1}\left\{v_{i}\right\} \cup \bigcup_{\substack{i=0, i \bmod 2=1}}^{n-2}\left\{u_{i}\right\} \cup \bigcup_{\substack{i=n-1, i \bmod 2=1}}^{n}\left\{u_{i}\right\} \\
& =F_{1,1}^{n} \cup \bigcup_{\substack{i=n, i \bmod 2=0}}^{n+1}\left\{v_{i}\right\} \cup \bigcup_{\substack{i=n-1, i \bmod 2=1}}^{n}\left\{u_{i}\right\} \\
& =F_{1,1}^{n} \cup\left\{v_{n+1}\right\} \cup\left\{u_{n}\right\}
\end{aligned}
$$

and

$$
\begin{aligned}
F_{1,2}^{n+1} & =\bigcup_{\substack{i=0, i \bmod 2=1}}^{n+2-2}\left\{v_{i}\right\} \\
& =\bigcup_{\substack{i=0,2 \\
i \bmod 2=1}}^{n-2}\left\{v_{i}\right\} \cup \bigcup_{\substack{i=n-1, i \bmod 2=1}}^{n}\left\{v_{i}\right\} \\
& =F_{1,2}^{n} \cup\left\{v_{n}\right\}
\end{aligned}
$$

Then

$$
\begin{aligned}
& F_{1,1}^{n+2} \cup F_{1,2}^{n+2} \\
= & F_{1,1}^{n} \cup F_{1,2}^{n} \cup\left\{v_{n+1}, u_{n}, v_{n}\right\} \\
= & F_{1,1}^{n} \cup F_{1,2}^{n} \cup T_{1, n}^{n+2}
\end{aligned}
$$

Since $G\left[T_{1, n}^{n+2}\right] \cong K_{1,3}$, then $G\left[T_{1, n}^{n+2}\right]$ is a tree.

By the induction hypothesis, $G\left[F_{1,1}^{n} \cup F_{1,2}^{n}\right]$ is a tree.

Tree $G\left[T_{1, n}^{n+2}\right]$ and tree $G\left[F_{1,1}^{n} \cup F_{1,2}^{n}\right]$ just have one common leaf vertex $v_{n-1}$, then $G\left[F_{1,1}^{n} \cup F_{1,2}^{n} \cup T_{1, n}^{n+2}\right]$ is a tree.

Combining induction step with basic step, $G\left[F_{1,2}^{n} \cup F_{1,2}^{n}\right]$ is a tree.

Thus, $G\left[V_{a}^{1}\left(M_{2 n}\right)\right]=G\left[F_{1,1}^{n} \cup F_{1,2}^{n}\right]$ is acyclic.

The lemma holds.

By Lemma 3.1 and Lemma 3.2, we obtain the Lemma 3.3 as follows.

Lemma 3.3. $V\left(M_{2 n}\right) \backslash V_{a}^{i}\left(M_{2 n}\right)$ is The feedback vertex set of $\mathrm{M}_{2 n}$ for $i \in[1,2]$.

\section{Feedback Number of $M_{2 n}$}

By reference [1], Beineke and Vandell prove a lower bound of general graphs $G(V, E)$ :

$$
f(G) \geq\left\lceil\frac{|E|-|V|+1}{\Delta-1}\right\rceil
$$

Lemma 4.1. Denote $f\left(M_{2 n}\right)$ as the feedback number of $M_{2 n}$, the lower bound of the feedback number is 


$$
f\left(M_{2 n}\right) \geq\left\lceil\frac{n+1}{2}\right\rceil
$$

Proof.

Since $\left|V\left(M_{2 n}\right)\right|=2 n,\left|E\left(M_{2 n}\right)\right|=3 n$ and $M_{2 n}$ is 3regular graph. Then, we obtain a lower bound as follows:

$$
f\left(M_{2 n}\right) \geq\left\lceil\frac{|E|-|V|+1}{\Delta-1}\right\rceil=\left\lceil\frac{3 n-2 n+1}{2}\right\rceil=\left\lceil\frac{n+1}{2}\right\rceil
$$

Lemma 4.2. Denote $f\left(M_{2 n}\right)$ as the feedback number of $M_{2 n}$, the upper bound of the feedback number is

$$
f\left(M_{2 n}\right) \leq\left\lceil\frac{n+1}{2}\right\rceil
$$

Proof. By Lemma 3.3, we have

$$
f\left(M_{2 n}\right) \leq V\left(M_{2 n}\right) \backslash V_{a}^{i}\left(M_{2 n}\right)
$$

Then

$$
\begin{aligned}
f\left(M_{2 n}\right) & \leq\left\{\begin{array}{l}
2 n-\left(n+\frac{n-1}{2}\right) \text { for odd } n \\
2 n-\left[(n-1)+\left(\frac{n}{2}-1\right)+1\right] \text { for even } n
\end{array}\right. \\
& =\left\lceil\frac{n+1}{2}\right\rceil
\end{aligned}
$$

By the Lemma 4.1 and Lemma 4.2, we obtain the feedback number of $M_{2 n}$ as follows.

Theorem1. For $n \geq 3$, the feedback number of $M_{2 n}$ is $f\left(M_{2 n}\right)=\left\lceil\frac{n+1}{2}\right\rceil$

\section{Acknowledgment}

The work was supported by NNSF of China (No.61802046) and Key Research Items in Natural Science Foundation of Liaoning (No.20170520327) and Doctoral Start up Fund of Dalian Ocean University (No.HDYJ201818).

\section{References}

1. L. W. Beineke, R. C. Vandell, Decycling graphs, J. Graph Theory, 25, 59-77, (1997).

2. I. Niven, H. S. Zuckerman, An Introduction to the Theory of Numbers ( 5 th ed.). John Wiley and Sons, New York, (1991).

3. I.Caragiannis, C. Kaklamanis, P. Kanellopoulos, New bounds on the size of the minimum feedback vertex set in meshes and butterflies. Information Processing Letters, 83, 75-80, (2002).

4. P. Festa, P. M. Pardalos, M. G. C. Resende, Feedback set problems. Handbook of Combinatorial
Optimization (D.-Z. Du, P.M. Pardalos eds.), Vol. A, Kluwer, Dordrecht, pp. 209, (1999).

5. V. Bafna, P. Berman, T. Fujito, A 2-approximation algorithm for the undirected feedback vertex set problem. SIAM J. Discrete Mathematics, 12, 289297, (1999).

6. S. Bau, L. W. Beineke, Z. Liu, G. Du, R. C. Vandell, Decycling cubes and grids. Utilitas Math., 59, 129137, (2001).

7. R. Bar-Yehuda, D. Geiger, J. S. Naor, R. M. Roth, Approximation algorithms for the feedback vertex set problem with applications to constraint satisfaction and Bayesian inference. SIAM J. Comput., 27, 942-959, (1998).

8. R. Focardi, F. L. Luccio, D. Peleg, Feedback vertex set in hypercubes. Information Processing Letters, 76 1-5, (2000).

9. Y. D. Liang, On the feedback vertex set in permutation graphs. Information Processing Letters, 52, 123-129, (1994).

10. F. L. Luccio, Almost exact minimum feedback vertex set in meshes and butterflies. Information Processing Letters, 66, 59-64, (1998)

11. G. W. Smith, Jr. and R. B. Walford, The identification of a minimal feedback vertex set of a directed graph. IEEE Trans. Circuits and Systems, 22, 9-15, (1975)

12. C.-C.Wang, E. L. Lloyd, M. L. Soffa, Feedback vertex sets and cyclically reducible graphs. J. Assoc. Comput. Mach., 32, 296-313, (1985).

13. F.-H. Wang, C.-J. Hsu, J.-C. Tsai. Minimal feedback vertex sets in directed splitstars. Networks, 45, 218223, (2005).

14. M. R. Garey, D. S. Johnson, Computers and Intractability, Freeman, San Francisco, CA, (1979).

15. R. Kralovic and P. Ruzicka, Minimum feedback vertex sets in shuffle-based interconnection networks Information Processing Letters, 86 (4) (2003), 191196.

16. J. C. Bermond and C. Peyrat, De Bruijn and Kautz networks: a competitor for the hypercube? In Hypercube and Distributed Computers (F. Andre and J. P. Verjus eds.). North-Holland: Elsevier Science Publishers, 1989, 278-293.

17. J.-M. Xu, Y.-Z. Wu, J. Huang, C.Yang, Feedback numbers of Kautz digraphs. Discrete Math., 307(13)(2007), 1589-1599.

18. J.-M. Xu, Topological Structure and Analysis of Interconnection Networks. Kluwer Academic Publishers, Dordrecht/Boston/London, 2001.

19. S.J.Zhang, X.R.Xu, C.Yin, N.Cao, Y.S.Yang, Feedback Numbers of Augmented Cubes AQn, Utilitas Mathematica, 97,183-192, (2015)

20. S.J.Zhang, X.R.Xu,C.Yin,et al. The feedback number of Knödel graph W3, n, ARS Combinatoria, 140, 397-409, (2018). 